\title{
PRELIMINARY STUDY ON THE DIATOM FLORA OF COASTAL PERIPHITIC ASSEMBLAGES OF THE RED SEA AND SUEZ GULF, EGYPT.
}

\author{
Ahmed M. El-Shahed \\ Botany Department, Faculty of Science, Minia University, 61519 Minia, Egypt
}

\begin{abstract}
Diatom flora of the coastal assemblages of the red Sea and Suez gulf in Egypt was investigated. 191 diatom taxa of diatoms were identified; 58 genera and 10 families. Naviculaceae (101 taxa), fragilariaceae (35 taxa), bacillariaceae (20 taxa and achnanthaceae (12 taxa) were the most common families. The data indicate that diatom populations of the study area appeared to be mainly dependent on the growth of certain genera viz; Mastogloia, Nitzschia, Amphora and Diploneis. Diatom assemblages were characteristic of tropical/ subtropical marine waters, however, a number of alloctonous diatom species were also recorded.
\end{abstract}

Key words: Marine diatoms, Periphyton, Coastal waters, Red Sea, Egypt

\section{Introduction}

The Red Sea is an intercontinental rift enclosed between Asia and Africa which is $1932 \mathrm{~km}$ long and $280 \mathrm{~km}$ wide. It is a unique environment since there are no permanent river inflows, rainfall is sparse, evaporation largely exceeds precipitation and seawater exchange with the Arabian Sea in the south is limited due to the very shallow sill of the Strait of Bab el Mandab (Seeberg-Elverfeldt, 2004). Due to high evaporation, values of sea surface salinity are high and increase from south to north up to $40 \%$ (Edwards, 1987).

Little is known about the algal flora of the coastal water of the Red Sea in Egypt. References dealing with this subject are restricted to the phytoplankton (Halim, 1969; Ibrahim, 1988; Zalat, 1997; El-Naggar et. al., 2002). Shaikh et al. (1986) and El-Naggar et al. (2002) found that diatoms were the most elaborated group forming phytoplankton communities of the Red Sea. Diatoms were also the main contributors of all marine sediments of the northern Red Sea (SeebergElverfeldt, 2004).

In Saudi Arabia, the coastal water of the Red Sea was also scarcely investigated for algal flora. Dowidar (1983) and Shaikh et al. (1986) dealt with the primary productivity of phytoplankton in relation to a number of ecological variables. Khalil and Ibrahim (1987) conduced a study on seasonal fluctuation of phytoplankton productivity. 
The present work deals with the diatom flora of the coastal periphytic assemblages of the Red Sea and Suez gulf in Egypt in an attempt towards the completion of knowledge on the Egyptian algal flora.

\section{Materials and Methods}

Sampling sites were located along the west coasts of Red Sea and Suez Gulf as well as the east coast of Suez Gulf. The area of study is almost limited by the latitudes $26^{\circ} 35^{\prime}-29^{\circ} 40^{\prime} \mathrm{N}$ and the longitudes $32^{\circ} 25^{\prime}-34^{\circ} 20^{\prime}$ E Ten sampling sites were chosen (Figure 1). Sampling sites were visited once during July and August 2005.

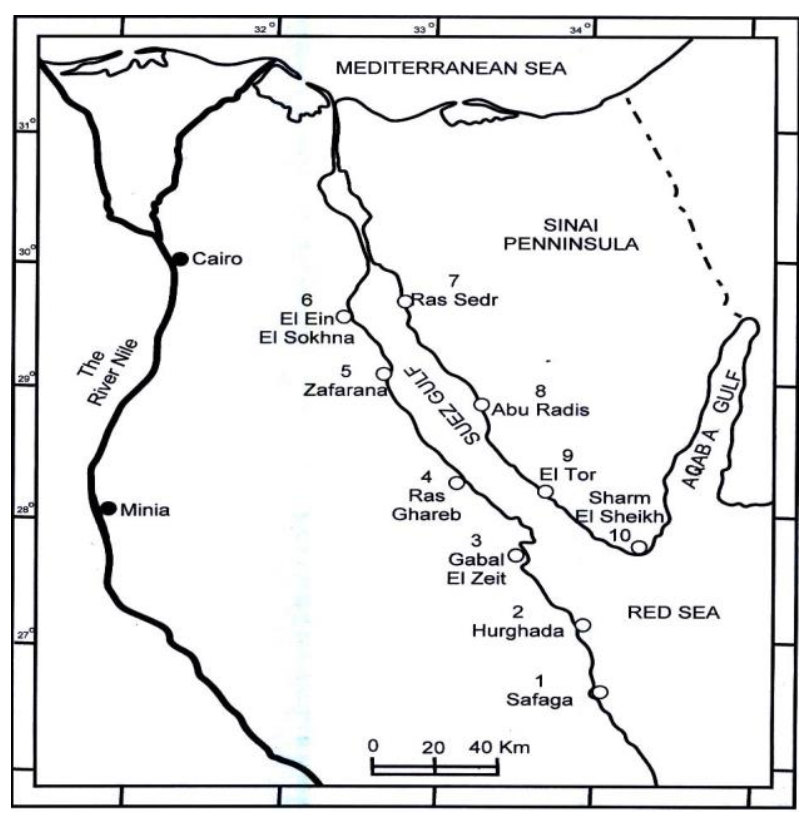

Figure (1): Map of the study area indicating sampling sites

Diatom samples were collected according to the standard methods reported by Prescott (1982), from solid objects found in water such as stones, mollusk shells, coral fragments, filamentous algae, seaweeds and aquatic plants. Samples were collected at well-illuminated areas at a depth up to $50 \mathrm{~cm}$. Each site was represented by three sampling places and samples were mixed together and treated as a single sample. Samples were preserved by $2 \%$ formaldehyde solution after collecting and were transported later to the laboratory.

Samples were treated in the laboratory for the removal of organic debris in water and the protoplast of diatom cells so as to keep clear the diatom valves (Wojtal and Sobczyk, 2006). Samples were kept in conc. HCl overnight, boiled 
for 10 min., rinsed several times with dist. water and then boiled in $33 \% \mathrm{H}_{2} \mathrm{O}_{2}$ with trace amounts of $\mathrm{KClO}_{3}$. After rinsing several times with dist. water, the clean diatom material was collected by decantation and suspended in 50\% ethanol. Examination with light microscopy was performed using a phase contrast microscope. Diatom specimens were photographed at $1000 \mathrm{X}$ magnification using an oil immersion lens.

For Scanning electron microscopy, aliquots of the digested samples were gently spread on cover slips, allowed to air-dry and were thereafter carbon-coated. Examination was performed using a Philips XL 30 ESEM scanning electron microscope.

Identification of diatoms was performed according to; Cleve-Euler (19511955), Krammer and Lange-Bertalot (1986, 1988, 1991a, b), Krammer (1992), Lange-Bertalot (1993), Metzeltin and Witkowski (1996), Witkowski et al., (2000). Relative abundance was based on counts of a given taxon in relation to the 500 total diatom valves that were counted for each sample. Taxa that attained $5 \%$ of the total counted valves were considered abundant; those with $2-5 \%$ were considered moderately abundant while taxa with less than $2 \%$ were considered rare (Wojtal and Sobczyk, 2006). Presence of taxa was calculated as percent of the number of sites in which a given taxon was recorded in relation to the total number of study sites.

\section{Results and Discussion}

The present results (Table 1) represent the diatom flora in the investigated area over a period from May to August 2005. The total number of diatom taxa (192) was composed of 58 genera and 10 families.

The data indicate that the reported diatom taxa can be considered characteristic for tropical/subtropical marine waters according to SeebergElverfeldt, (2004). Number of taxa recorded for individual sampling sites was not consistent. The largest number of taxa was observed at sites 9 and 10. These sites are more likely to be the least subjected to oil pollution and it seems that tourism in Sharm El-Sheikh (site 10) does not produce about drastic changes to the environment. On the other hand, the least number of species was recorded at site 8 near the town Abu Rdis which is a big center of oil industry that may have adverse effects on aquatic life.

The data indicate that populations of most of the constituent species were small where ca $62 \%$ of the taxa was considered rare according to the used scale of abundance. Moderately abundant and abundant populations were recorded for about $30 \%$ and $7 \%$ of the constituent taxa, respectively. The Red Sea, especially in its northern part, has been frequently reported as an oligotrophic environment that was found to be stratified for most of the year. The depth of thermocline varies seasonally where it exceeds $200 \mathrm{~m}$ in winter and becomes shallow (3060m) during spring and summer (Seeberg-Elverfeldt, 2004). 
Table (1):Distribution and relative abundance of the recorded diatom taxa in the studied sites. Abundance of populations is denoted by,+++ and +++ as rare, moderately abundant and abundant, respectively.

\begin{tabular}{|c|c|c|c|c|c|c|c|c|c|c|c|c|c|}
\hline Sites & 1 & 2 & 3 & 4 & 5 & 6 & 7 & 8 & 9 & 10 & 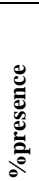 & 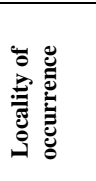 & 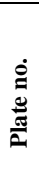 \\
\hline Taxa & ++++ & ++++ & +++ & +++ & ++++ & ++++ & +++ & +++ & +++ & ++++ & & & \\
\hline \multicolumn{14}{|c|}{ Family: Thalassiosiraceae Lebour 1930} \\
\hline \multicolumn{14}{|c|}{ Genus: Cyclotella (Kützing) Brebisson1838 } \\
\hline C. ocellata Pantocsek & & & + & & & + & & & + & & 30 & $3,6,9$ & $\begin{array}{c}\text { VII } \\
\text { I }\end{array}$ \\
\hline \multicolumn{14}{|c|}{ Genus: Stephanodiscus Ehrenberg 1845} \\
\hline $\begin{array}{l}\text { S. tephanodiscus } \\
\text { hantzschii Grun. }\end{array}$ & & & & & & + & & & & + & 20 & 6,10 & \\
\hline \multicolumn{14}{|c|}{ Family: Coscinodiscaceae Kützing 1844} \\
\hline \multicolumn{14}{|c|}{ Genus: Coscinodiscus Ehrenberg 1838} \\
\hline C. nitidus Greg. & & & & & & & & & ++ & & 10 & 9 & I \\
\hline C. subvelatus Kütz. & & & & & & & & & ++ & & 10 & 9 & I \\
\hline \multicolumn{14}{|c|}{ Genus: Endictya Ehrenberg 1845} \\
\hline $\begin{array}{l}\text { Endictya oceanica } \\
\text { Ehr. }\end{array}$ & & & & + & & & & & & & 10 & 4 & \\
\hline \multicolumn{14}{|c|}{ Family: Hemidiscaceae Henedy 1964} \\
\hline \multicolumn{14}{|c|}{ Genus: Actinocyclus Ehrenberg 1837} \\
\hline A. ochotensis Jouse & & & & & & & & & +++ & & 10 & 9 & I \\
\hline A.subtilis(Greg.)Ralfs & + & & & & & ++ & & & + & & 30 & $1,6,9$ & I \\
\hline \multicolumn{14}{|c|}{ Genus: Bacteriastrum Shadbolt 1845} \\
\hline B. delicatulum $\mathrm{Cl}$. & & & & & & & & & & + & 10 & 10 & \\
\hline \multicolumn{14}{|c|}{ Genus: Stictodiscus Greville 1861} \\
\hline $\begin{array}{lr}\text { S. parallelus } \mathrm{v} . \\
\text { balnearis } \\
\text { Peragallo }\end{array}$ & & & & & & & & + & & & 10 & 8 & \\
\hline \multicolumn{14}{|c|}{ Family: Biddulphiaceae Henedy 1931} \\
\hline \multicolumn{14}{|c|}{ Genus: Biddulphia Gray 1821} \\
\hline B. pulchella Gray & & + & & & & & & & +++ & & 20 & 2,9 & I, II \\
\hline \multicolumn{14}{|c|}{ Genus: Odontella Kützing 1844} \\
\hline O.aurita (Lyngb.) Ag. & & ++ & & & & ++ & ++ & & & & 30 & $2,6,7$ & $\begin{array}{c}\text { VII } \\
\text { I }\end{array}$ \\
\hline \multicolumn{14}{|c|}{ Genus: Terpsinö̈ Ehrenberg 1843} \\
\hline $\begin{array}{l}\text { T. americana (Bailey) } \\
\text { Ralfs }\end{array}$ & & & & & & & & & + & & 10 & 9 & \\
\hline \multicolumn{14}{|c|}{ Genus: Triceratium Ehrenberg 1841} \\
\hline T. paralellum Greville & & & & & & & + & & & + & 20 & 7,10 & \\
\hline T. reticulatum Ehr. & & & & + & & & & & & & 10 & 4 & I \\
\hline \multicolumn{14}{|c|}{ Family: Fragilariaceae Hustedt 1930} \\
\hline \multicolumn{14}{|c|}{ Genus: Ardissonia De Notaris 1870} \\
\hline $\begin{array}{l}\text { A. cristallina } \\
\text { (Ag.) Grun. }\end{array}$ & & & & & ++ & & & ++ & & & 20 & 5,8 & \\
\hline $\begin{array}{l}\text { A. formosa } \\
\text { (Hantzsch) Grun. }\end{array}$ & & & & ++ & & & & & + & & 20 & 4,9 & $\begin{array}{l}\text { III, } \\
\text { IX }\end{array}$ \\
\hline $\begin{array}{l}\text { A. fulgens (Greville) } \\
\text { Grun. }\end{array}$ & & & & + & & & & & & & 10 & 4 & \\
\hline $\begin{array}{l}\text { A. robusta (Ralfs) De } \\
\text { Notaris }\end{array}$ & & & & & & & & & + & & 10 & 9 & \\
\hline \multicolumn{14}{|c|}{ Genus: Climacosphenia Ehrenberg 1843} \\
\hline C. moniligera Ehr. & + & & + & & +++ & & & & & + & 40 & $1,3,5,10$ & IV \\
\hline \multicolumn{14}{|c|}{ Genus: Fragilaria Lyngbye 1819} \\
\hline $\begin{array}{l}\text { F.beroliensis(Lemm.) } \\
\text { Lange-Bertalot }\end{array}$ & & & & & & & & ++ & & & 10 & 8 & IX \\
\hline 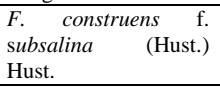 & & ++ & & & & & & & & & 10 & 2 & IX \\
\hline $\begin{array}{l}\text { F. fasciculata (Ag.) } \\
\text { Lange Bertalot }\end{array}$ & & & & +++ & & & & & & & 10 & 4 & \\
\hline
\end{tabular}

Egyptian J. of Phycol. Vol. 7(2), 2006

$-188-$ 
Preliminary Study on The Diatom Flora of Coastal Periphitic Assemblages of .......

\begin{tabular}{|c|c|c|c|c|c|c|c|c|c|c|c|c|c|}
\hline $\begin{array}{l}F . \quad \text { leptostauron v. } \\
\text { dubia (Grun.) Hust. }\end{array}$ & & & & & & ++ & & + & + & & 30 & $6,8,9$ & IX \\
\hline $\begin{array}{l}\text { F. leptostauron } v . \\
\text { martyii (Herib.) } \\
\text { Lange Bertalot }\end{array}$ & ++ & + & ++ & ++ & & & + & & + & + & 70 & $\begin{array}{c}1-4,7,9 \\
10\end{array}$ & \\
\hline $\begin{array}{l}\text { F. pinnata v. pinnata } \\
\text { Ehr. }\end{array}$ & & & ++ & & ++ & & & & & & 20 & 3,5 & IX \\
\hline $\begin{array}{l}F . \\
\text { Witkowski \& Lanotensis } \\
\text { Bertalot }\end{array}$ & & & & & & & & & & ++ & 10 & 10 & IX \\
\hline $\begin{array}{l}F . \quad \text { ulna (Nitzsch) } \\
\text { Lange Bertalot }\end{array}$ & & + & + & + & + & & & + & + & + & 70 & $2-5,8-10$ & \\
\hline $\begin{array}{lrr}\begin{array}{l}F . \quad \text { ulna } \\
\text { (Kütz.) }\end{array} & \text { acus } \\
\text { Bertalot } & & \text { Lange } \\
\end{array}$ & & & & + & & & & & & & 10 & 4 & \\
\hline \multicolumn{14}{|c|}{ Genus: Fragilariopsis Hustedt 1913} \\
\hline Fragilariopsis sp. 1 & & & & & & & & & & + & 10 & 10 & VII \\
\hline F. sp. 2 & & & & & & & & & & + & 10 & 10 & VII \\
\hline \multicolumn{14}{|c|}{ Genus: Grammatophora Ehrenberg 1840} \\
\hline $\begin{array}{l}\text { G. angulosa v. } \\
\text { islandica (Ehr.) Grun. }\end{array}$ & & & & & & & & & & + & 10 & & \\
\hline G. maxima Grun. & & & & & & & & & & + & 10 & & \\
\hline G. undulata Ehr. & & & & & & & & & & + & $\mathbf{1 0}$ & & \\
\hline \multicolumn{14}{|c|}{ Genus: Hyalosynedra Williams \& Round 1986} \\
\hline $\begin{array}{l}\text { H. laevigata (Grun.) } \\
\text { Williams \& Round }\end{array}$ & + & +++ & & + & & & + & & +++ & +++ & 60 & $\begin{array}{c}1,2,4,7,9, \\
10\end{array}$ & IX \\
\hline \multicolumn{14}{|c|}{ Genus: Licmophora Agardh 1827} \\
\hline L. gracilis(Ehr.)Grun. & & +++ & & & & + & + & & & & 30 & $2,6,7$ & \\
\hline $\begin{array}{l}\text { L. g. v. anglica } \\
\text { (Kütz.) Peragallo }\end{array}$ & & & & + & & & & & & & 10 & 4 & $\begin{array}{l}\text { VI, } \\
\text { IX }\end{array}$ \\
\hline $\begin{array}{l}\text { L. paradoxa (Lyngb.) } \\
\text { Ag. }\end{array}$ & & & & & & & & & +++ & + & 20 & 9,10 & \\
\hline L. remulus Grun. & & + & & & ++ & & & & +++ & +++ & 40 & $2,5,9,10$ & IV \\
\hline \multicolumn{14}{|c|}{ Genus: Opephora Petit 1883} \\
\hline $\begin{array}{l}\text { O. mutabilis (Grun.) } \\
\text { Sabbe\& Vyverman }\end{array}$ & & & & & & & & & + & & 10 & 9 & \\
\hline \multicolumn{14}{|c|}{ Genus: Plagiogramma Greville 1859} \\
\hline P. rhombicum Hust. & & + & & & & ++ & ++ & & & & 30 & $2,6,7$ & \\
\hline $\begin{array}{l}\text { P. pulchellum } v . \\
\text { pygmaeum (Greville) } \\
\text { Peragallo }\end{array}$ & & ++ & & & & & & & ++ & & 20 & 2,9 & VII \\
\hline \multicolumn{14}{|c|}{ Genus: Protoraphis Simonsen 1970} \\
\hline $\begin{array}{l}\text { Protoraphis atlantica } \\
\text { Gibson }\end{array}$ & & & & & & & & & & +++ & 10 & 10 & \\
\hline \multicolumn{14}{|c|}{ Genus: Rhabdonema Kützing 1844} \\
\hline R. minutum Kütz. & & & & & & & & & & + & $\mathbf{1 0}$ & 10 & \\
\hline R. adriaticum Kütz. & & & & + & & & & & & & 10 & 4 & \\
\hline \multicolumn{14}{|c|}{ Genus: Rhaphoneis Ehrenberg 1844} \\
\hline $\begin{array}{l}\text { R. amphicerus (Ehr.) } \\
\text { Ehr. }\end{array}$ & & & & & & & & & + & & 10 & 9 & \\
\hline \multicolumn{14}{|c|}{ Genus: Striatella Agardh 1832} \\
\hline $\begin{array}{l}\text { S. unipunctata } \\
\text { (Lyngb.) Ag. }\end{array}$ & & & & & & & + & & & & 10 & 7 & \\
\hline \multicolumn{14}{|c|}{ Genus: Tabellaria Ehrenberg 1844} \\
\hline $\begin{array}{l}\text { T. flocculosa (Roth) } \\
\text { Kütz. }\end{array}$ & & + & & & & & & & & & 10 & 2 & \\
\hline \multicolumn{14}{|c|}{ Genus: Trachysphenia Petit 1877} \\
\hline T. australis Petit & & & & & & & & & & ++ & $\mathbf{1 0}$ & 10 & \\
\hline $\begin{array}{l}\text { T. australis } \mathrm{v} . \\
\text { rostellata Hust. }\end{array}$ & & & & & + & ++ & & + & + & & 40 & $5,6,8,9$ & \\
\hline \multicolumn{14}{|c|}{ Family: Achnanthaceae Henedy 1964} \\
\hline \multicolumn{14}{|c|}{ Genus: Achnanthes Bory 1822} \\
\hline A. amoena Hust. & & ++ & & & & & & & & & 10 & 2 & $\mathbf{X}$ \\
\hline A. brevipes & & & & + & + & & + & & & + & 40 & $4,5,7,10$ & \\
\hline $\begin{array}{l}\text { A. cuneata (Grun.) } \\
\text { Grun. }\end{array}$ & & & & & & & & & & + & 10 & 10 & \\
\hline A. exciliata Giffen & & & & & & & & ++ & & & 10 & 8 & \\
\hline $\begin{array}{l}\text { A. pseudogroenlandica } \\
\text { Hendey }\end{array}$ & & & & & & & + & & & + & 20 & 7,10 & \\
\hline
\end{tabular}

Egyptian J. of Phycol. Vol. 7(2), 2006

$-189-$ 
Ahmed M. El-Shahed

\begin{tabular}{|c|c|c|c|c|c|c|c|c|c|c|c|c|c|}
\hline C. hauniensis Witkowki & & & & & ++ & & & & & & 10 & 5 & $\mathbf{X}$ \\
\hline C. hoffmannii Simonsen & & & & & + & & & & & & 10 & 5 & \\
\hline $\begin{array}{l}\text { C. krammerii Lange- } \\
\text { Bertalot \& Metzeltin }\end{array}$ & & & & & & & & & ++ & + & 20 & 9,10 & $\mathbf{x}$ \\
\hline C. pellucida Grun. & & & & & & & & & & ++ & 10 & 10 & \\
\hline $\begin{array}{l}\text { C. scutellum } \mathrm{v} \text {. } \\
\text { scutellum } \text { Ehr. }\end{array}$ & + & & & & & & & & & + & 20 & 1,10 & \\
\hline $\begin{array}{l}\text { C. s. v. speciosa } \\
\text { (Greg.) Cl. }\end{array}$ & & ++ & & & & & & & & & 10 & 2 & $\mathbf{x}$ \\
\hline $\begin{array}{l}\text { C. stauroneiformis } \\
\text { (W. Smith) Okuno }\end{array}$ & & & & & & & & & & + & 10 & 10 & \\
\hline \multicolumn{14}{|c|}{ Family: Naviculaceae Bessey 1907} \\
\hline \multicolumn{14}{|c|}{ Genus: Amphora Ehreberg 1840} \\
\hline $\begin{array}{l}\text { A. bigibba v. } \\
\text { interrupta (Grun.) } \\
\text { Grun. }\end{array}$ & & & & + & & & & & & & 10 & 4 & \\
\hline $\begin{array}{l}\text { A. cymatophora } \\
\text { Cholnoky }\end{array}$ & + & & & & & & & & & & 10 & 1 & II \\
\hline $\begin{array}{l}\text { A. delicatissima } \\
\text { Krasske }\end{array}$ & & & & & ++ & ++ & & ++ & & & 30 & $5,6,8$ & $\mathbf{X I}$ \\
\hline A. egregia Ehr. & & & & & & & + & & & & 10 & 7 & II \\
\hline A. exciliata Giffen & & & ++ & & & ++ & & & & & 20 & 3,6 & XI \\
\hline A. gracilis $\mathrm{W}$. Smith & & & & + & & & & & & & 10 & 4 & II \\
\hline A. holsatica Hust. & ++ & & & + & & & & & ++++ & & 30 & $1,4,9$ & II \\
\hline $\begin{array}{l}\text { A. holsaticoides } \\
\text { Nagumo\& Kobayasi }\end{array}$ & ++ & & & & & & + & & & + & 30 & $1,7,10$ & II \\
\hline A. laevissima Greg. & + & + & & & & & & & & & 20 & 1,2 & \\
\hline A. obtusa Greg. & ++ & & & & & & & & & & 10 & 1 & \\
\hline A. ostrearia Breb. & & & & & & & & & ++ & & 10 & 9 & \\
\hline A. ovalis (Kütz.) Kütz. & & & & & & & & & + & & 10 & 9 & \\
\hline A. proteus Greg. & ++ & & & & ++ & & & & & & 20 & 1,5 & II \\
\hline A. rhombica Kitton & & & & & & & & & ++ & & 10 & 9 & \\
\hline A. securicula Peragallo & & & & & & & ++ & & & & 10 & 7 & \\
\hline A. spectabilis Greg. & & & & & & & & ++ & ++ & & 20 & 8,9 & II \\
\hline \multicolumn{14}{|c|}{ Genus Anomoeoneis Pfitzer 1871} \\
\hline $\begin{array}{l}\text { A. sphaerophora } \\
\text { (Kütz.) Pfitzer }\end{array}$ & & & & & & & + & & & & 10 & 7 & $\mathbf{V}$ \\
\hline \multicolumn{14}{|c|}{ Genus: Berkeleya Greville 1827} \\
\hline $\begin{array}{l}\text { B. scopulorum (Breb.) } \\
\text { Cox }\end{array}$ & & & +++ & & ++ & & & & & & 20 & 3,5 & \\
\hline \multicolumn{14}{|c|}{ Genus: Caloneis Cleve 1894} \\
\hline C. alpestris (Grun.) Cl. & & & & & & & & & + & & 10 & 9 & \\
\hline $\begin{array}{l}\text { C. amphisbaena } \\
\text { (Bory) } \mathrm{Cl} \text {. }\end{array}$ & & & & + & & & & & & & 10 & 4 & III \\
\hline C. elongata (Grun.) $\mathrm{Cl}$. & & & & & & & & & + & & 10 & 9 & \\
\hline $\begin{array}{l}\text { C. excentrica (Grun.) } \\
\text { Cl. }\end{array}$ & & & & & & & & & ++ & & 10 & 9 & $\mathbf{v}$ \\
\hline $\begin{array}{l}\text { C. liber (W. Smith) } \\
\text { Cl. }\end{array}$ & ++ & & & & + & & + & & ++ & & 40 & $1,5,7,9$ & \\
\hline $\begin{array}{l}\text { C. linearis (Grun.) } \\
\text { Boyer }\end{array}$ & ++ & + & & & & ++ & & & & & 30 & $1,2,6$ & \\
\hline \multicolumn{14}{|c|}{ Genus: Craticula Grunow 1868} \\
\hline $\begin{array}{l}\text { C. cuspidata (Kütz.) } \\
\text { D.G. Mann }\end{array}$ & & & & & & & & & & ++ & 10 & 10 & $\mathbf{x}$ \\
\hline \multicolumn{14}{|c|}{ Genus: Diploneis Ehrenberg 1844} \\
\hline D. aestiva (Donk.) Cl. & & & & & ++ & & & & & & 10 & 5 & III \\
\hline $\begin{array}{l}\text { D. bomboides } \\
\text { (Schmidt) Cl. }\end{array}$ & & & & & & & & & + & & 10 & 9 & III \\
\hline D. bombus Ehr. & & & & & & & + & & & + & 20 & 7,10 & III \\
\hline D. crabro Ehr. & + & & & + & + & & + & & & & 40 & $1,4,5,7$ & \\
\hline $\begin{array}{l}\text { D. incurvata (Greg.) } \\
\text { Cl. }\end{array}$ & & & & & & ++ & & & ++ & + & 30 & $6,9,10$ & \\
\hline $\begin{array}{l}\text { D. littoralis } v . \\
\text { littoralis (Donkin) } \mathrm{Cl} \text {. }\end{array}$ & & & & & & & & & & + & 10 & 10 & III \\
\hline $\begin{array}{l}\text { D. notabilis (Greville) } \\
\text { Cl. }\end{array}$ & & & & & ++ & & & & & & 10 & 5 & \\
\hline D. rex Droop & & & & & & & & & + & & 10 & 9 & \\
\hline $\begin{array}{l}\text { D. smithii v. smithii } \\
\text { (Breb.) Cl. }\end{array}$ & ++ & + & & & & & & & + & & 30 & $1,2,9$ & III \\
\hline D. suborbicularis $v$. & & & & & + & & & & & & 10 & 5 & \\
\hline
\end{tabular}

Egyptian J. of Phycol. Vol. 7(2), 2006

-190 - 
Preliminary Study on The Diatom Flora of Coastal Periphitic Assemblages of .......

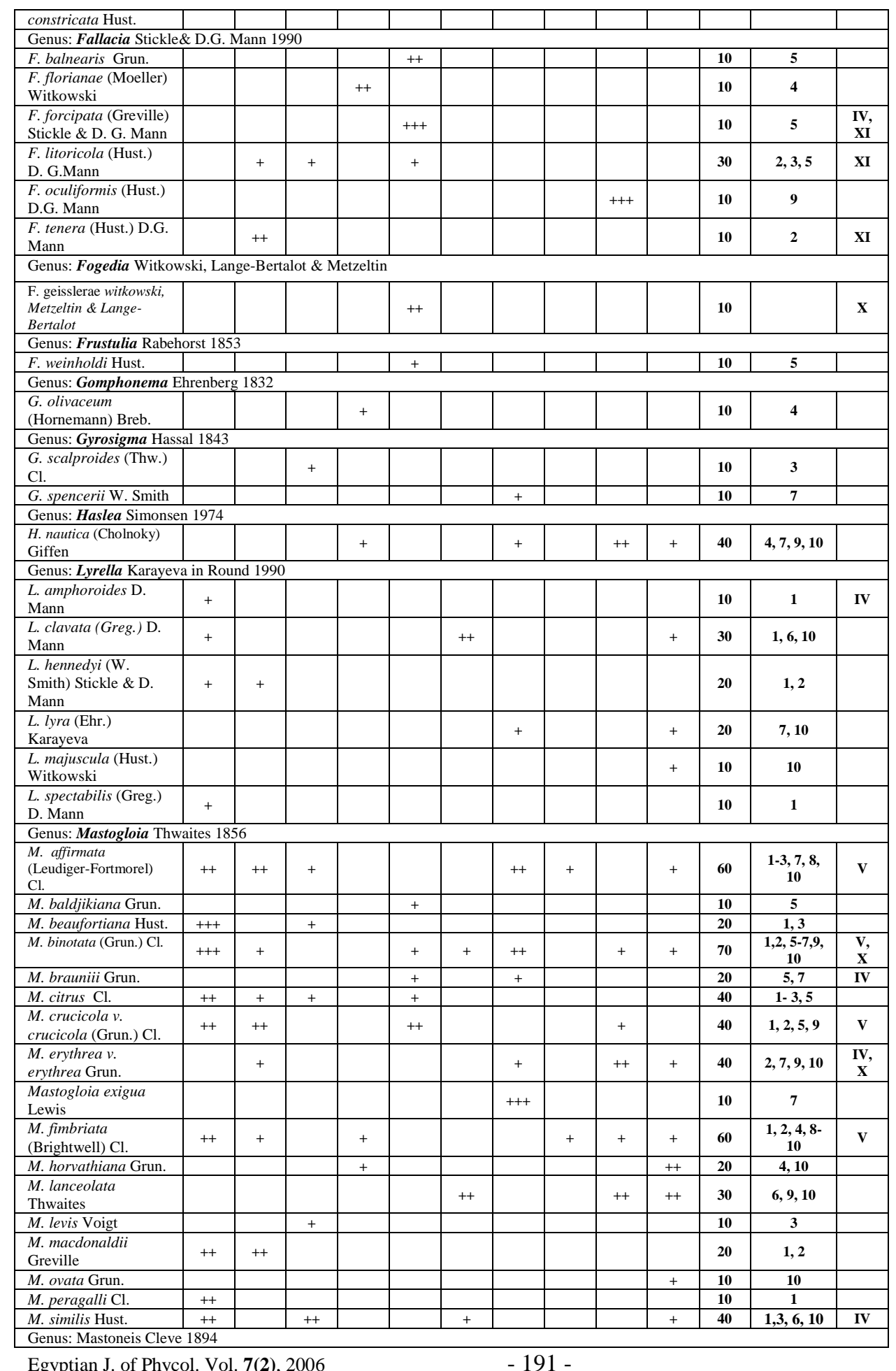


Ahmed M. El-Shahed

\begin{tabular}{|c|c|c|c|c|c|c|c|c|c|c|c|c|c|}
\hline $\begin{array}{l}\text { Mastoneis biformis } \\
\text { (Grun.) Cl. }\end{array}$ & ++ & & + & & & & + & & & & 30 & $1,3,7$ & VI \\
\hline \multicolumn{14}{|c|}{ Genus: Navicula Bory 1822} \\
\hline N. borneoensis Hust. & & & & & & & & & & + & 10 & 10 & \\
\hline $\begin{array}{l}\text { N. cryptocephala } \\
\text { Kütz. }\end{array}$ & & & & & & & & + & & & 10 & 8 & \\
\hline $\begin{array}{l}\text { N. digitatoradiata } \\
\text { (Greg.) Ralfs }\end{array}$ & +++ & & + & & + & & & & & & 30 & $1,3,5$ & III \\
\hline$N$. flagellifera (Hust.) & & & & & & & & + & & & 10 & 8 & \\
\hline N. pagophila Grun. & & & + & & & & & & & & 10 & 3 & \\
\hline N. palpebrialis Breb. & & & & & & & + & & & & $\mathbf{1 0}$ & 7 & \\
\hline N. pavillardii Hust. & & & & & & & + & & & & $\mathbf{1 0}$ & 7 & \\
\hline $\begin{array}{l}\text { N. tripunctata }(\mathrm{O} . \\
\text { Müller) Bory }\end{array}$ & & & ++ & & & + & & + & & & 30 & $3,6,8$ & \\
\hline \multicolumn{14}{|c|}{ Genus: Parlibellus Cox 1988} \\
\hline $\begin{array}{l}\text { P. delognei (Van } \\
\text { Heurck) Cox }\end{array}$ & & & & + & & & & & & & 10 & 4 & \\
\hline \multicolumn{14}{|c|}{ Genus: Petroneis Stickle \& D.G. Mann 1990} \\
\hline $\begin{array}{l}\text { Petroneis glaciales } v \text {. } \\
\text { hudsonii } \mathrm{Cl} \text {. }\end{array}$ & + & & & & & & & & & & 10 & 1 & VI \\
\hline $\begin{array}{l}\text { P. humerosa (Breb.) } \\
\text { Stickle \& D. Mann }\end{array}$ & + & & & & & & & & & & 10 & 1 & VI \\
\hline \multicolumn{14}{|c|}{ Genus: Pinnularia Ehrenberg 1843} \\
\hline $\begin{array}{l}\text { P. stauroptera } \\
\text { (Grun.) Cl. }\end{array}$ & & & & & & & & ++ & & & 10 & 8 & VII \\
\hline \multicolumn{14}{|c|}{ Genus: Plagiotropis Pfitzer 1871} \\
\hline P. neovitrea Paddock & ++ & & & + & & & + & & & + & 40 & $1,4,7,10$ & \\
\hline $\begin{array}{l}\text { P. pusilla (Greg.) } \\
\text { Kuntze }\end{array}$ & & & & & + & & & & & & 10 & 5 & \\
\hline \multicolumn{14}{|c|}{ Genus: Pleurosigma W. Smith 1852} \\
\hline $\begin{array}{l}\text { P. angualtum (Quek.) } \\
\text { Cl. }\end{array}$ & & & & & & & & & & ++ & 10 & 10 & \\
\hline $\begin{array}{l}\text { P. elongatum } \mathrm{W} . \\
\text { Smith }\end{array}$ & & & & & & & & & + & & 10 & 9 & \\
\hline $\begin{array}{l}\text { P. naviculaceum } \\
\text { Breb. }\end{array}$ & & & & & + & & & & & & 10 & 5 & \\
\hline P. normanii Ralfs & & & & & & & & & & ++ & $\mathbf{1 0}$ & 10 & \\
\hline P. praelongum $\mathrm{Cl}$. & + & & & & & & & & & & $\mathbf{1 0}$ & 1 & VI \\
\hline P. salinarum Grun. & + & & + & & & ++ & & & & & 30 & $1,3,6$ & \\
\hline $\begin{array}{l}\text { P. strigosum } \mathrm{W} . \\
\text { Smith }\end{array}$ & + & & & & & ++ & & & + & & 30 & $1,6,9$ & \\
\hline P. s. v. latum $\mathrm{Cl}$. & & & & & & & + & & & & $\mathbf{1 0}$ & 7 & VI \\
\hline \multicolumn{14}{|c|}{ Genus: Seminavis D. G. Mann 1990} \\
\hline Seminavis sp. 1 & + & & ++ & + & + & & + & & & + & 60 & $1,3-5,10$ & II \\
\hline Semnavis sp. 2 & & ++ & + & & + & & +++ & & +++ & & $\mathbf{5 0}$ & $\begin{array}{c}2,3,5,7 \\
9\end{array}$ & II \\
\hline \multicolumn{14}{|c|}{ Genus: Stauroneis Ehrenberg 1843} \\
\hline S. anceps Ehr. & & & & & & & & & & + & 10 & 10 & \\
\hline S. groenlandica Östr. & & ++ & & & & & & & & & 10 & 2 & \\
\hline S. plicata Brockmann & & & & & & & + & & & & 10 & 7 & \\
\hline \multicolumn{14}{|c|}{ Genus: Trachyneis Petit in Folin \& Pierre 1877} \\
\hline T. aspera (Ehr.) Cl. & & + & + & + & + & ++ & ++++ & & & + & 70 & $2-7,10$ & VI \\
\hline T. clepsydra Donkin & & & & & + & & & & & & 10 & 5 & \\
\hline T. velata A. Schmidt & & & & & & & & & + & & 10 & 9 & \\
\hline \multicolumn{14}{|c|}{ Family: Bacillariaceae Ehrenberg 1840} \\
\hline \multicolumn{14}{|c|}{ Genus: Bacillaria Gmelin 1791} \\
\hline $\begin{array}{l}\text { B. socialis (Greg.) } \\
\text { Ralfs }\end{array}$ & & & & + & & & & & & & 10 & 4 & \\
\hline B. paradoxa Gmelin & & & & & & & & ++ & & & 10 & 8 & \\
\hline $\begin{array}{l}\text { B. paxillifer }(\mathrm{O} \\
\text { Müller) Hendey }\end{array}$ & & & & & & & & & & + & 10 & 10 & \\
\hline \multicolumn{14}{|c|}{ Genus: Nitzschia Hassal 1845} \\
\hline $\begin{array}{l}\text { N. acicularis (Kütz.) } \\
\text { W. Smith }\end{array}$ & & & & & & & & & + & & 10 & 9 & \\
\hline $\begin{array}{l}\text { N. angularis W. } \\
\text { Smith }\end{array}$ & & & & & & & & & +++ & & 10 & 9 & \\
\hline $\begin{array}{l}\text { N. constricta (Kütz.) } \\
\text { Ralfs }\end{array}$ & & & & + & & & & & & & 10 & 4 & \\
\hline $\begin{array}{l}\text { N. dealpina Lange } \\
\text { Bertalot \& Hofmann }\end{array}$ & & & & & & ++ & & & & & 10 & 6 & XII \\
\hline
\end{tabular}

Egyptian J. of Phycol. Vol. 7(2), 2006

- 192 - 


\begin{tabular}{|c|c|c|c|c|c|c|c|c|c|c|c|c|c|}
\hline $\begin{array}{l}\text { N. dissipata (Kütz.) } \\
\text { Ralfs }\end{array}$ & & & & & & & ++ & & & & 10 & 7 & \\
\hline N. distans Grun. & & & & & & & & & + & & 10 & 9 & \\
\hline $\begin{array}{l}\text { N. lanceolata W. } \\
\text { Smith }\end{array}$ & & & & & & & & & & + & 10 & 10 & \\
\hline N. lorenziana Grun. & & & + & & & & & & & & 10 & 3 & \\
\hline $\begin{array}{l}\text { N. marginulata } \mathrm{v} . \\
\text { marginulata Grun }\end{array}$ & ++ & & & +++ & + & & + & + & + & +++ & 70 & $\begin{array}{c}1,4,5,7- \\
10\end{array}$ & VII \\
\hline $\begin{array}{l}N . \text { panduriformis } \mathrm{v} . \\
\text { continua Grun. }\end{array}$ & & & & & & & + & & & & 10 & 7 & XII \\
\hline $\begin{array}{l}N \text {. panduriformis } \mathrm{v} \text {. } \\
\text { panduriformis } \mathrm{De} \\
\text { Toni }\end{array}$ & & & & & + & ++ & & & + & & 30 & $5,6,9$ & VII \\
\hline $\begin{array}{l}\text { N. panduriformis v. } \\
\text { lata (Witt) Grun. }\end{array}$ & & & & & & & ++ & & & & 10 & 7 & \\
\hline N. pellucida $\mathrm{Cl}$. & & & + & & & & & & & & 10 & 3 & \\
\hline N. reversa W. Smith & & & & & & & & & + & & 10 & 9 & \\
\hline $\begin{array}{l}\text { N. scalpelliformis } \\
\text { (Grun.) Grun. }\end{array}$ & & & & & & & & & & ++ & 10 & 10 & VII \\
\hline N. ventricosa Kitton & & & & & & & & & & + & 10 & 10 & VII \\
\hline $\begin{array}{l}\text { N. vermicularis } \\
\text { (Kütz.) Hantzsch }\end{array}$ & & & + & & & & & & & & 10 & 3 & VII \\
\hline \multicolumn{14}{|c|}{ Family: Epithemiaceae Grunow 1860} \\
\hline \multicolumn{14}{|c|}{ Genus: Rhopalodia O. Müller 1895} \\
\hline $\begin{array}{l}\text { R. musculus (Kütz.) } \\
\text { O. Müller }\end{array}$ & & & ++ & & + & ++ & & & & + & 40 & $3,5,6,10$ & $\mathbf{V}$ \\
\hline R. pacifica Krammer & & & & & & & & ++ & & & 10 & 8 & $\mathbf{X I}$ \\
\hline \multicolumn{14}{|c|}{ Family: Surirellaceae Henedy 1937} \\
\hline \multicolumn{14}{|c|}{ Genus: Campylodiscus Ehrenberg 1840} \\
\hline C. decorus Breb. & ++ & & + & & & + & & & & & 30 & $1,3,6$ & \\
\hline \multicolumn{14}{|c|}{ Genus: Stenopterobia Brebisson 1875} \\
\hline $\begin{array}{l}\text { S. curvula (W. Smith) } \\
\text { Krammer }\end{array}$ & & & + & & & & & & & & 10 & 3 & \\
\hline \multicolumn{14}{|c|}{ Genus: Surirella Turpin 1828} \\
\hline $\begin{array}{l}\text { S. ceylanensis v. } \\
\text { oblongestriata } \text { Hust. }\end{array}$ & & & & & & & & & ++ & & 10 & 9 & \\
\hline S. fastosa (Ehr.) Kütz. & + & & & & & + & & & + & + & 40 & $1,6,9,10$ & $\mathbf{V}$ \\
\hline S. lata W. Smith & & & & & & & + & & & & 10 & 7 & \\
\hline Surirella $\mathrm{sp} 1$ & + & & & & & & & & & & 10 & 1 & $\mathbf{V}$ \\
\hline Surirella sp. 2 & & & & & + & + & & & + & & 30 & $5,6,9$ & $\mathbf{V}$ \\
\hline Unknown diatom no. 1 & & & & & + & & & & ++++ & & 20 & 5,9 & XII \\
\hline Unknown diatom no. 2 & ++ & & & & ++ & & & & & + & 30 & $1,6,10$ & XII \\
\hline Number of taxa/ site & 42 & 29 & 28 & 29 & 39 & 27 & 38 & 18 & 54 & 58 & & & \\
\hline
\end{tabular}

This may explain the scarcity of populations in the herein samples that could be attributed to the scarcity of nutrients. Consumption of dissolved nutrients especially nitrates and phosphates by aquatic plants including algae, and the difficulty to compensate their concentrations in water by replenishment from bottom sediments, that is dictated by thermal stratification, lead to depletion of these important nutrients from water (Boney, 1989). Some authors reported increase in population size at certain localities as a consequence of contamination with domestic pollutants. El-Naggar et al., (2002) attributed increase in population size to enrichment of water with $\mathrm{N}$ and $\mathrm{P}$, the elements frequently reported as limiting nutrients (Boney, 1989). In the present investigation, it was taken in consideration to choose sampling sites far away as much as possible from pollution sources so as to report almost natural diatom assemblages.

Relatively a few number of taxa attained high presence values $(50 \%$ or more) whereas the majority of taxa (123 taxa) showed lower values of presence referring to low frequency of the constituent taxa. The present communities were Egyptian J. of Phycol. Vol. 7(2), 2006 $-193-$ 
characterized by a number of differential species that showed high fidelity to the communities of the investigated area viz; Fragilaria leptostauron v. matyii, F. ulna, Hyalosynedra laevigata, Achnanthes brevipes, Diploneis crabro, Mastogloia affirmata, M. binotata, M. fimbriata, Seminavis spp., Trachyneis aspera and Nitzschia marginulata v. marginulata (Plates II, V, VI, VII, IX and $\mathrm{X})$. None of these taxa were reported in the literature cited because most of the research was concerned with phytoplankton, however, most of them were included in the species lists for sediments of the Red Sea in its northern part (Seeberg-Elverfeldt, 2004) and in Lake Timsah (Zalat 1997).

Some taxa which are not typical marine residents and are rather characteristic of brackish water were recorded such as Cyclotella ocellata, Stephanodiscus hantzschii, Amphora ovalis, Anomoeoneis sphaerophora, Caloneis amphisbaena, Gomphonema olivaceum, Gyrosigma scalproides, G. spencerii, Navicula cryptocephala, N. tripunctata, Stauroneis anceps, and Nitzschia dissipata (Plates III and VIII). These taxa sporadically occurred and had small population size. They seem to be alloctonous species that were introduced to the environment through discharge through different anthropogenic activities (Zalat 1997).

In conclusion, diatom assemblages recorded to the studied area were characteristic of tropical/ subtropical marine waters that mostly appeared in small populations. These assemblages comprised a number of alloctonous diatom species that are not true marine forms.

\section{Acknowledgements}

I'm greatly indebted to Dr Jolanta Piatek, Szafer Institute of Botany, Polish Academy of Science, for her unfailing help concerning Scanning Electron Microscopy. I'm also grateful to Mr Yasser Hefaina, Travel Ways Egypt Co., for financial support of the journey for collecting samples.

\section{REFERENCES}

Boney, A. D. (1989). Phytoplankton. Edward Arnold Pub., London, New York, Melbourne, Aucland. 118 pp.

Cleve-Euler, A. (1951-1955). Die Diatomeen von Schweden und Finnland. K. Svenska Vet. Akad. Handl. Serie 4, 2/1: 1- 163 (1951); 3/3: 1-153 (1952); 4/1: 1-158 (1953); 4/5: 1-225 (1953); 5/4: 1-232 (1955).

Dowidar, N. M. (1983). Primary production in the central Red Sea off Jeddah. Bull. Nat. Inst. Oceanogr. and Fish. A.R.E., 9: 160- 170.

Edwards, F. J. (1987). Climate and oceanography. In: Edwards A. J. and Head S. M. (eds), Key environments, Red Sea. Pergamon Press, Oxford, 4570pp.

El-Naggar, A.; Osman, M.; El-Sherif, Z. and Nassar, M. (2002). Phytoplankton and seaweeds of Suez Gulf (from Red Sea) in relation to 
some physico-chemical factors, oil and sewage pollution. Bull. Fac. Sci. Assiut Univ., 31(I-D):77- 104.

Halim, Y. (1969). Plankton of the Red Sea. Oceanogr. Mar. Biol. Ann. Rev., 7:231- 275.

Hustedt, F. (1930). Die Kieselalgen. In: Rabenhorst (ed.) Kryptogamen Flora von Deutschland, Osterrisch und der Schweiz, 7(1): 920 pp.

Ibrahim, E. A. (1988). Observation on the distribution of phytoplankton in Foul Bay, Red Sea. Bull. Nat. Inst. Oceanogr. and Fish. ARE, 14(1):91- 103.

Khalil, A. N. and Ibrahim, A. M. M. (1987). A seasonal study of the surface phytoplankton of the Red Sea, North of Jeddah. Arab. Gulf J. Sci. Res., 6:189-204.

Krammer, K. (1992). Pinnularia eine monographie der europäischen taxa. In: Bibliotheca Diatomologica, Band 26, J. Kramer, Berlin, Stuttgart. 352pp.

Krammer, K. and Lange-Bertalot, H. (1986). Bacillariophyceae.1. Teil: Naviculaceae. In: Ettl, H., Gerloff, J., Heying, H. and Mollenhauer, D. (eds): Süßwasserflora Mitteleuropa 2/1, Pub. G. Fischer Verlag, Stuttgart, New York, 876 pp.

Krammer, K. and Lange-Bertalot, H. (1988). Bacillariophyceae. 2. Teil: Bacillariaceae, Epithemiaceae, Surirellaceae. In: Ettl, H., Gerloff, J., Heying, H. and Mollenhauer, D. (eds): Süßwasserflora Mitteleuropa 2/2, Pub. G. Fischer Verlag, Stuttgart- New York, 596 pp.

Krammer, K. and Lange-Bertalot, H. (1991a). Bacillariophyceae.3.Teil: Centrales, Fragilriaceae, Eunotiaceae. In: Ettl, H., Gerloff, J., Heying, H. and Mollenhauer, D. (eds): Süßwasserflora Mitteleuropa 2/1, Pub. G. Fischer Verlag, Stuttgart- Jena, 576 pp.

Krammer, K. and Lange-Bertalot, H. (1991b). Bacillariophyceae.4.Teil: Achnanthaceae, Kritische Ergänzungen zu Navicula (Lineolatae) und Gomphonema, Gesamtliteraturverzeichnis. In: Ettl, H., Gerloff, J., Heying, H. and Mollenhauer, D. (eds): Süßwasserflora Mitteleuropa 2/1, Pub. G. Fischer Verlag, Stuttgart- Jena, 437 pp.

Lange-Bertalot, H. (1993). 85 nue taxa, und über 100 weitere neu definierte taxa ergänzend zur Süßwasser von Mitteleuropa Vol. 2/1-4. In: Bibliotheca Diatomologica. Band 27. J. Kramer, Berlin, Stuttgart. 454 pp.

Metzeltin D. and Witkowski A. (1996). Diatomeen der Bären-Insel, Süßwasser und marine Arten. In: Lange-Bertalot H. (eds): Iconographia diatomologica, annotated diatom micrographs. Vol. 4 Taxonomy. Koeltz Scientific Books, Königstein, Germany. 287 pp.

Prescott, G. W. (1982). How to know freshwater algae. The pictured key series. Brown W. C. Com. Publ., Dubuque, Iowa, 293 pp.

Seeberg-Elverfeldt, I. A. (2004). Laminated diatomaceous sediments of the Red Sea, their composition and significance as recorders of abrupt changes 
in productivity and circulation during the Late Quaternary. $\mathrm{Ph}$. D. Thesis, Bremen University, Germany, 127 pp.

Shaikh, E. A.; Roff, G. C. and Dowidar, N. M. (1986). Phytoplankton ecology and production in the Red Sea off Jeddah, Saudi Arabia. Marine Biology, 92:405-416.

Silva, P. C. (1962). Classification of algae. In: Lewin R. A. (eds): Physiology and Biochemistry of algae. New York Academic Press, Appendix A, 827837 pp.

Simonsen, R. (1979). The diatom system: Leads in phylogeny. Bacillaria, 2:972.

Witkowski, A.; Lange-Bertalot, H. and Metzeltin, D. (2000). Diatom flora of marine coasts I. Gantner Verlag Pub. Köngenstein. 925 pp.

Wojtal, A. and Sobczyk, L. (2006). Composition and structure of epilithic diatom assemblages on stones of different size in a small calcareous stream (S Poland). Arch. Hydrobiol. Suppl. 162/ Algol. Stud., 119:105124.

Zalat, A. (1997). Distribution of Holocene diatoms and silicoflagellates in bottom sediments of the Lake Timsah, Suez Canal area, Egypt. Egyptian J. Geol., 41(1):103-128.

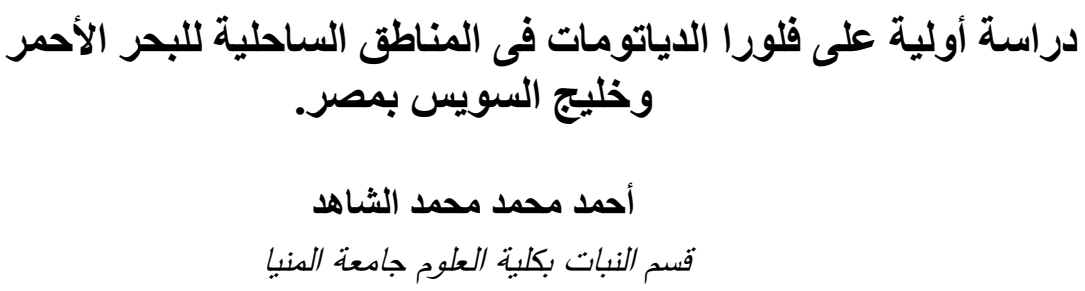

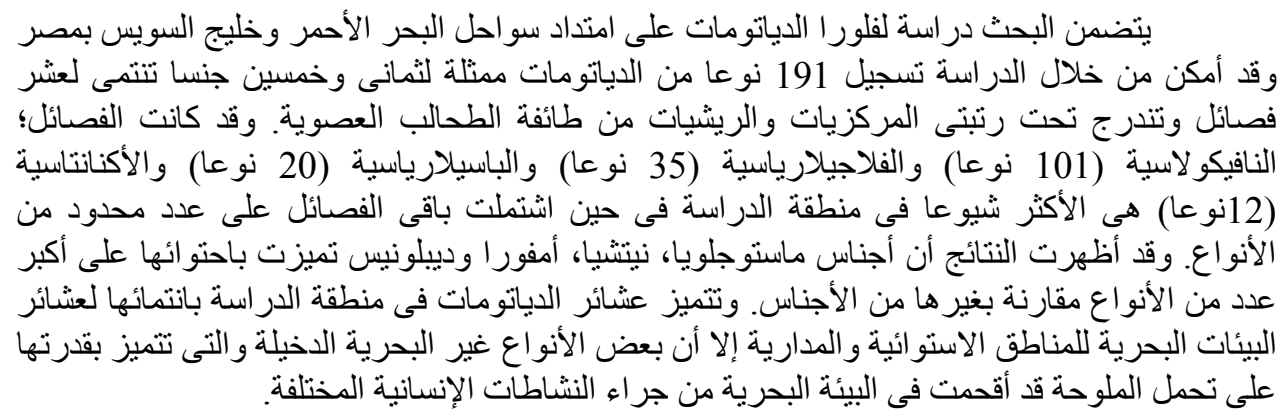


Preliminary Study on The Diatom Flora of Coastal Periphitic Assemblages of ......

\section{Plate(I)}
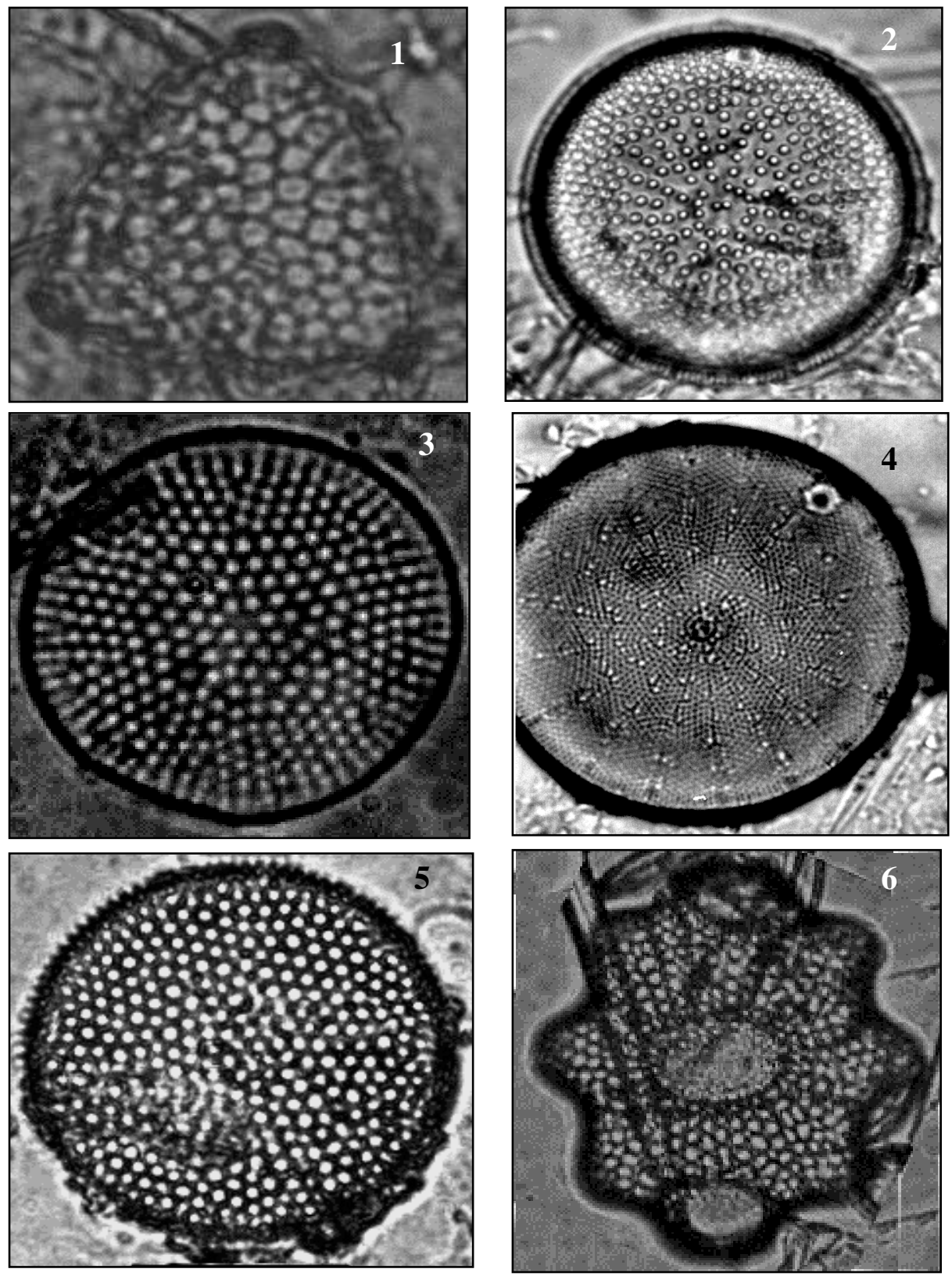

Figures 1-6; 1- Triceratium reticulatum Ehr. 2, Actinocyclus ochotensis Jouse, 3- A. subtilis (Greg.) Ralfs, 4- Coscinodiscus nitidus Greg., 5- C. subvelatus Greg., 6Biddulphia pulchella Gray (middle part of valve face). 


\section{Plate (II)}
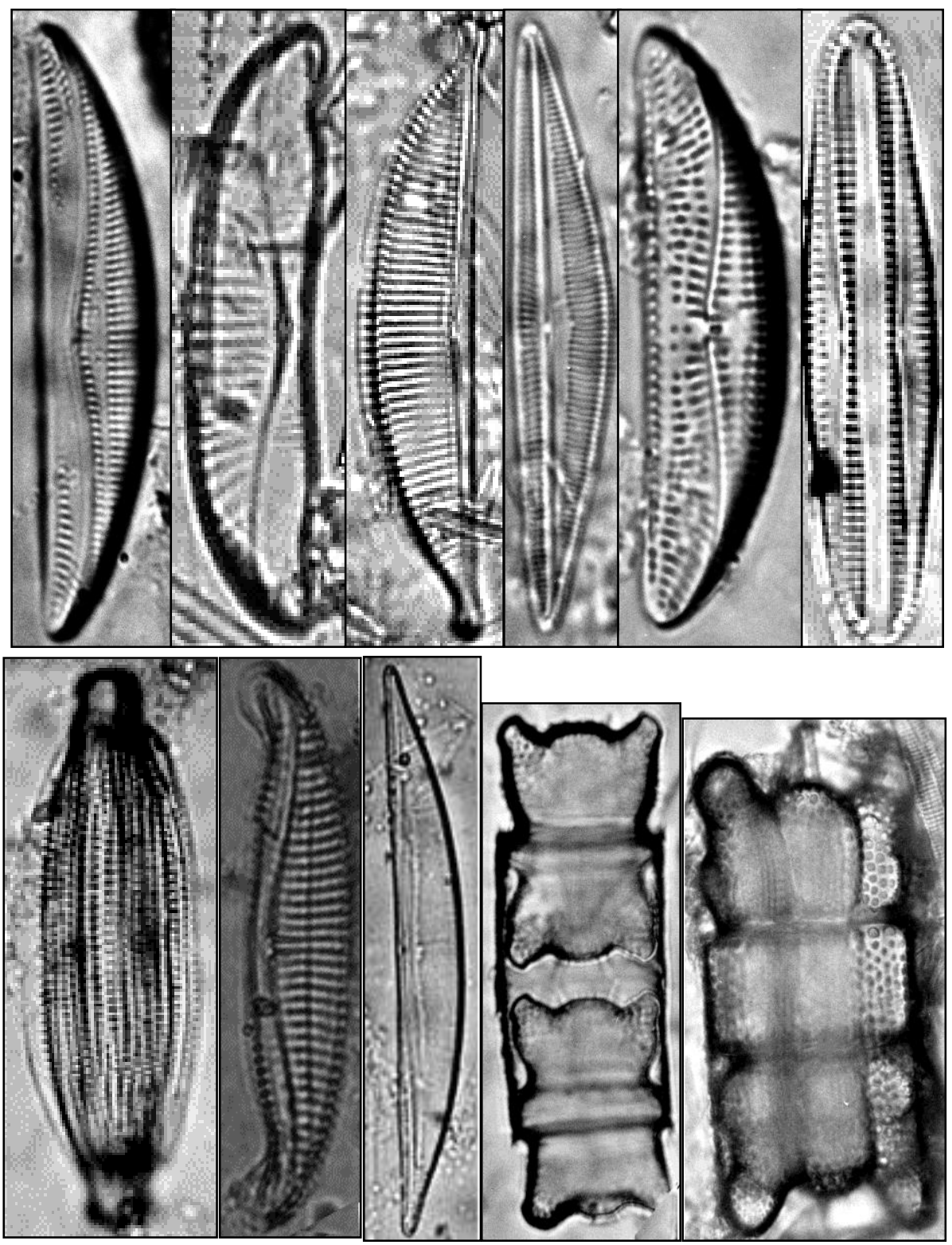

Figures 1-11; 1- Amphora gracilis W. Smith, 2- A. spectabilis Greg., 3- Amphora holsaticoides Nagumo \& Kobayasi, 4-, Seminavis sp., 5- Amphora proteus Greg., 6- A. cymatophora Cholnoky, 7- Amphora holsatica Hust., 8- A. egregia Ehr., 9- Seminavis sp.2, 10- Odontella aurita (Lyngb.) Ag., 11- Biddulphia pulchella Gray. 


\section{Plate (III)}
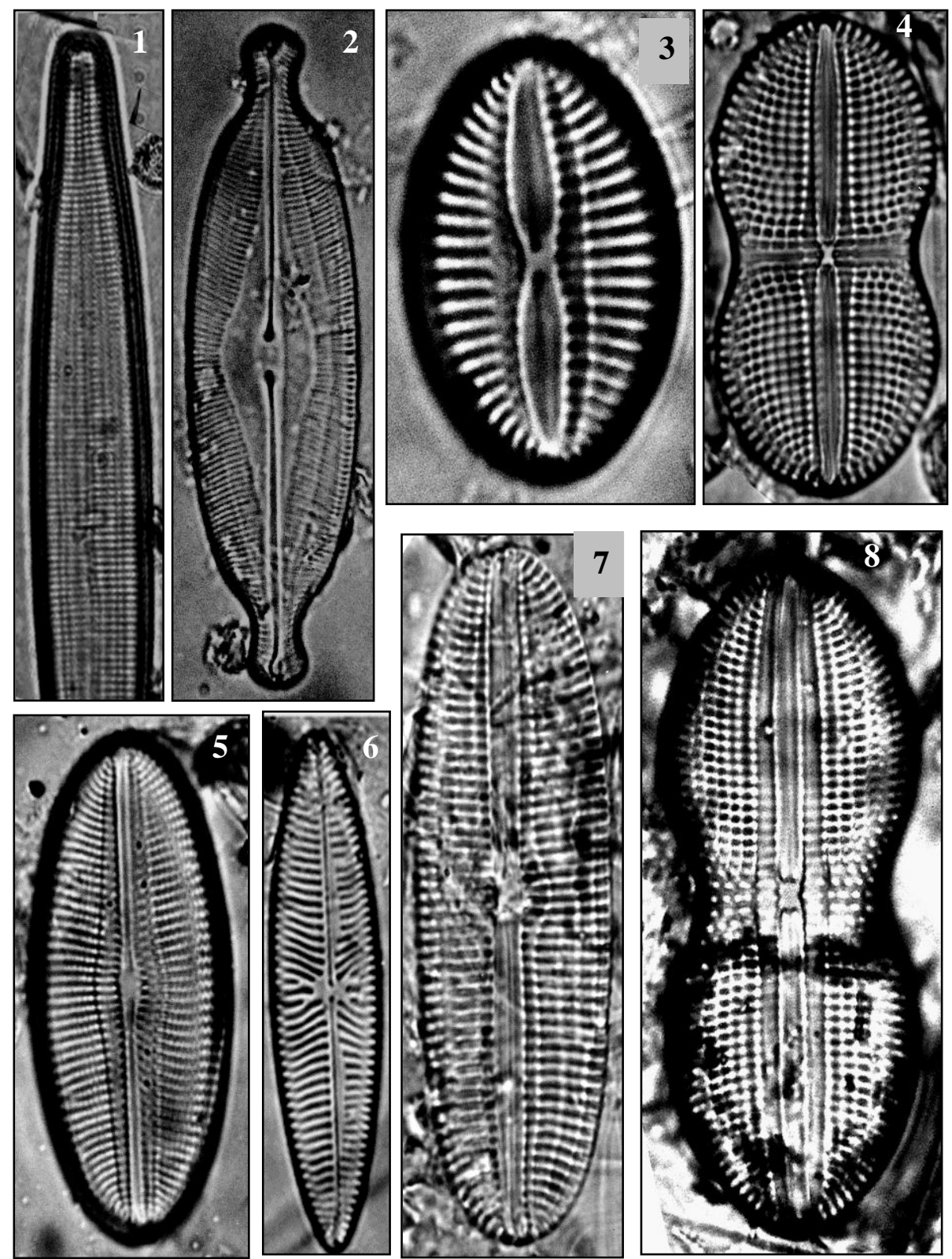

Figures 1-8; 1- Ardissonia formosa (Hantzsch) Grun, 2- Caloneis amphisbaena (Bory) Cl. 3- Diploneis smithii v. smithii (Breb.) Cl., 4- D. bombus Ehr., 5- D. litoralis v. litoralis (Donk.) Cl., 6- Navicula digitatoradiata (Greg.) Ralfs, 7- D. aestiva (Donk.) Cl., 8- D. bomboides (Schmidt) Cl. 


\section{Plate (IV)}
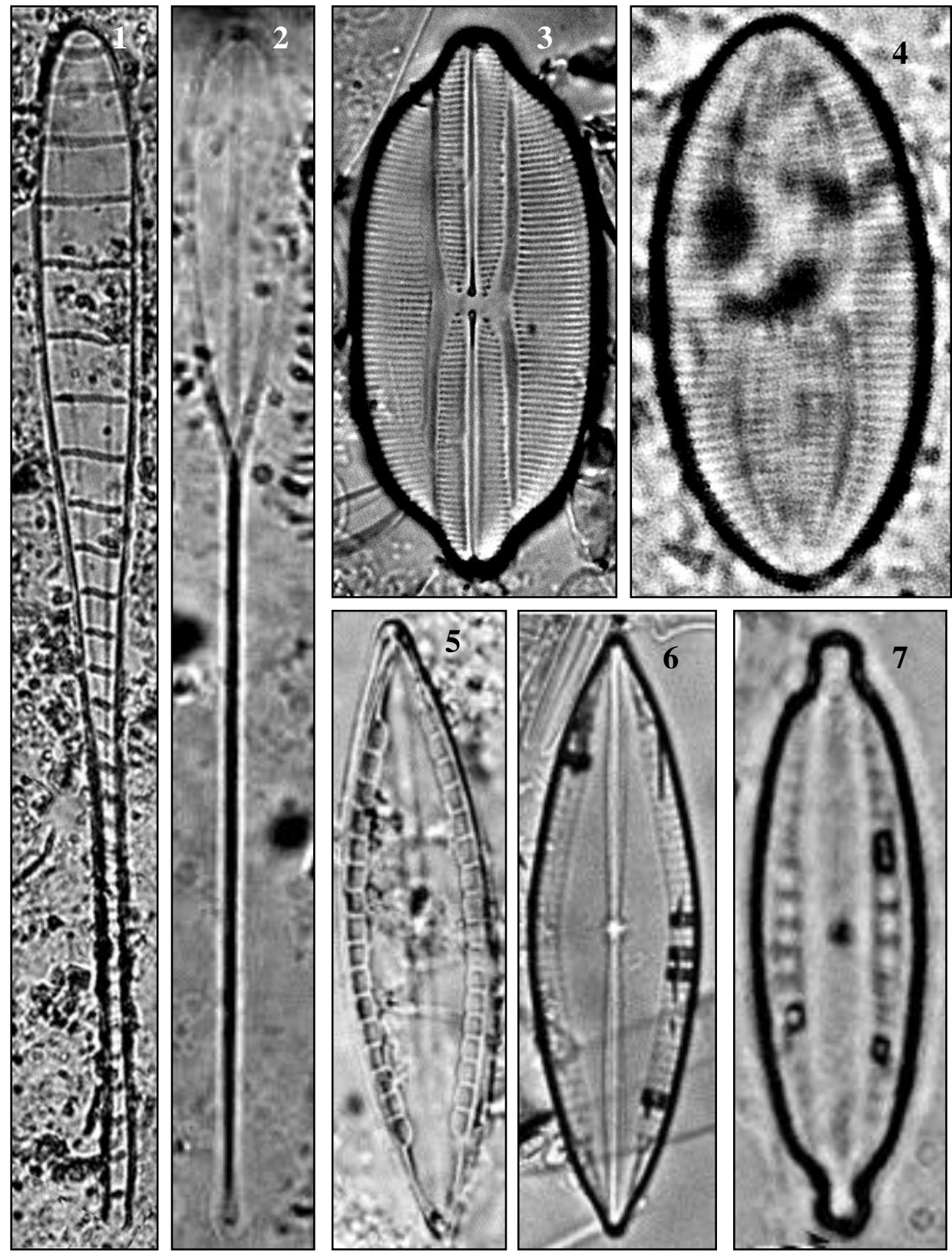

Figures 1-7; 1- Climacosphenia moniligera Ehr. 2, Licmophora remulus Grun., 3Lyrella amphoroides D. Mann, 4- Fallacia forcipata (Grev.) Stickle \& D. G. Mann, 5Mastogloia braunii Grun., 6- M. erythrea v. erythrea Grun., 7- M. similis Hust. 


\section{$\operatorname{Plate}(\mathbf{V})$}
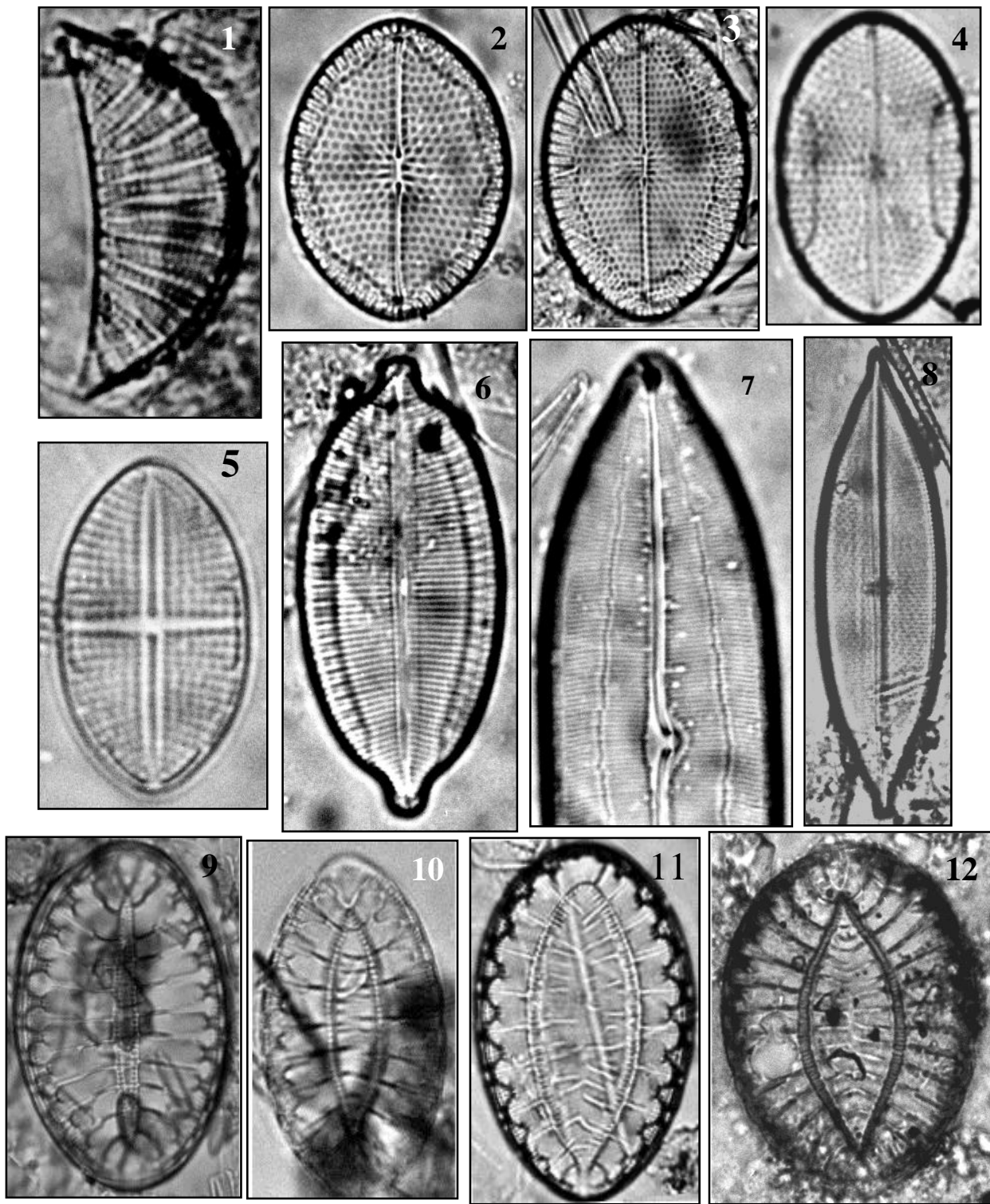

Figures1-12; 1- Rhopalodia musculus (Kütz.) O. Müller, 2, 3- Mastogloia fimbriata (Brightwell) Cl., 4- M. binotata (Grun.) Cl., 5- M. crucicola v. crucicola (Grun.) Cl., 6- M. affirmata (Leudiger-Fortmorel) Cl., 7-Caloneis excentrica (Grun.) Cl., 8Anomoeoneis sphaerophora (Kütz.) Pfitzer, 9, 10- Surirella fastusa (Ehr.) Kütz. 11- S. sp 1, 12- S. sp.2. 


\section{Plate (VI)}
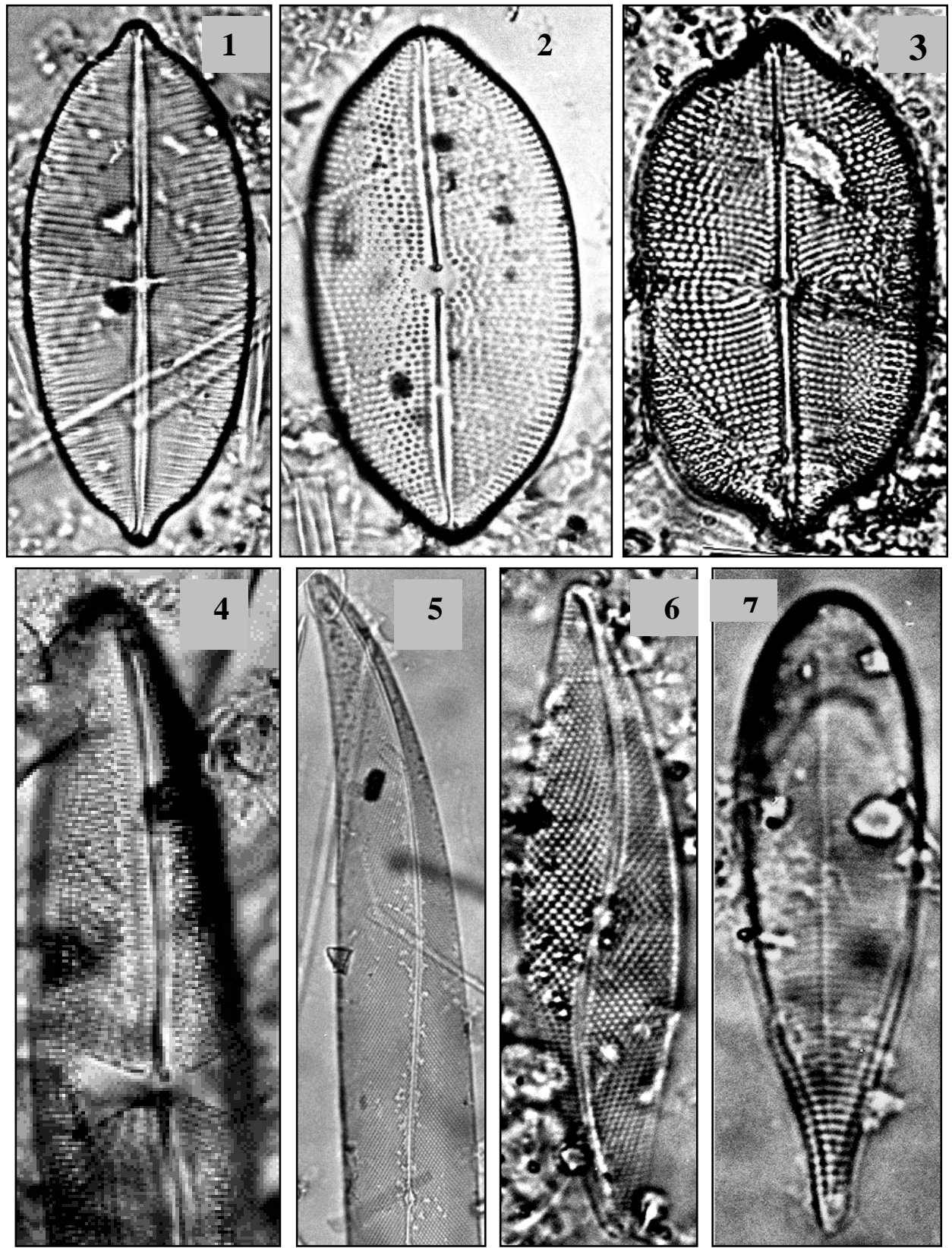

Figures 1-7; 1- Mastoneis biformis (Grun.) Cl., 2- Petroneis glaciales v. hudsonii Cl., 3- $P$. humerosa (Breb.) Stickle \& D.G. Mann, 4- Trachyneis aspera (Ehr.) Cl. 5Pleurosigma praelongum Cl., 6- P. strigosum v. latum Cl., 7- Licmophora gracilis v. anglica (Kütz.) Peragallo. 


\section{Plate (VII)}
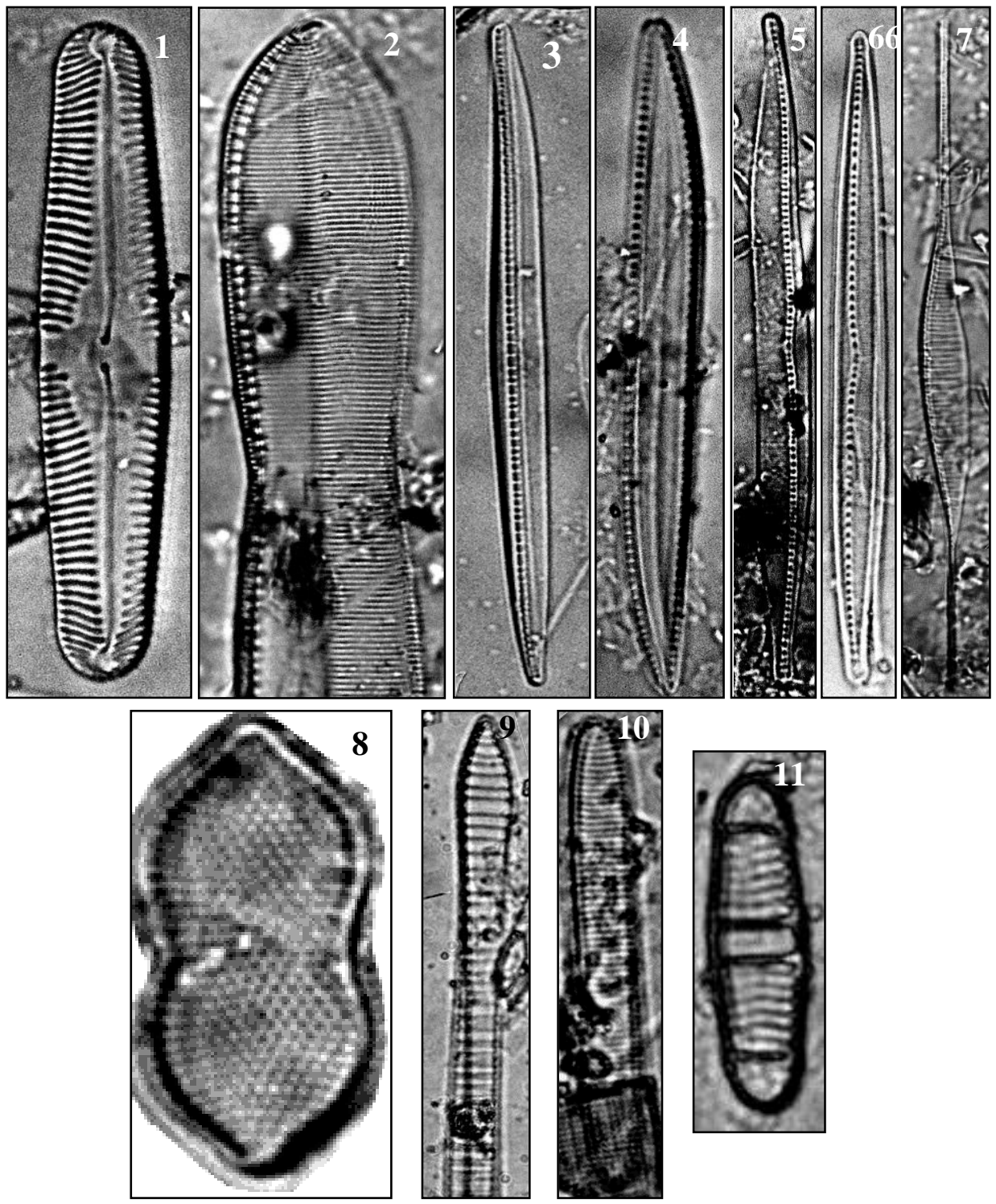

Figures 1-11; 1- Pinnularia stauroptera (Grun.) Cl., 2- Nitzschia marginulata v. marginulata Grun., 3, 4- N. vermicularis (Kütz.) Hantzsch, 5, 6- $N$. scalpelliformis Grun., 7- N. ventricosa Kitton, 8- N. panduriformis v. panduriformis De Toni, 9Fragilariopsis sp.1, 10- F. sp.2, 11- Plagiogramma pulchella v. pygmaeum (Grev.) Peragallo. 


\section{Plate (VIII)}
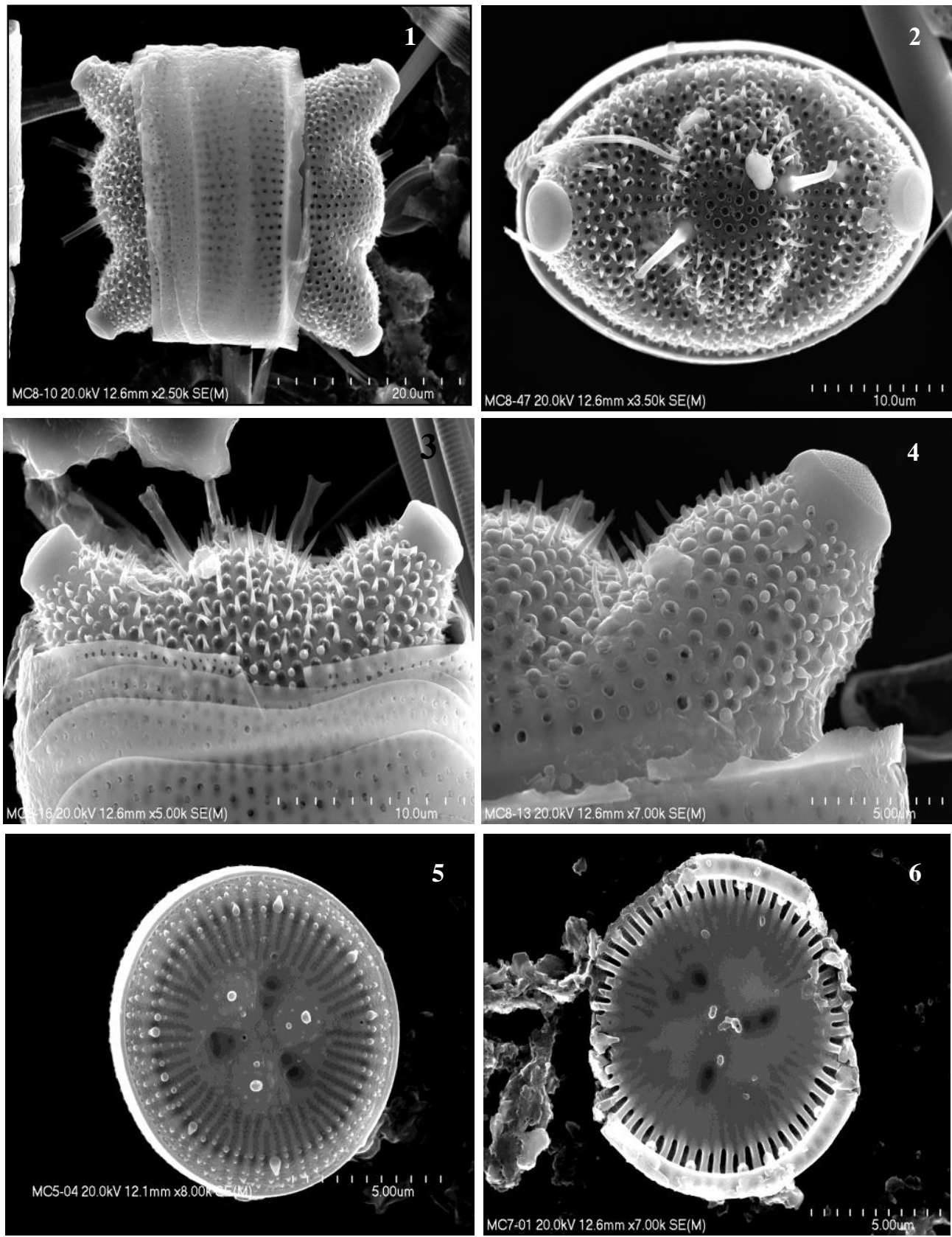

Figures 1-6; 1- 4 Odontella aurita (Lyngb.) Ag., 1, whole cell, 2- middle part of the valve face, 3,4 spines and elevations on the valve face, 5, 6-Cyclotella ocellata Pantocsek. 

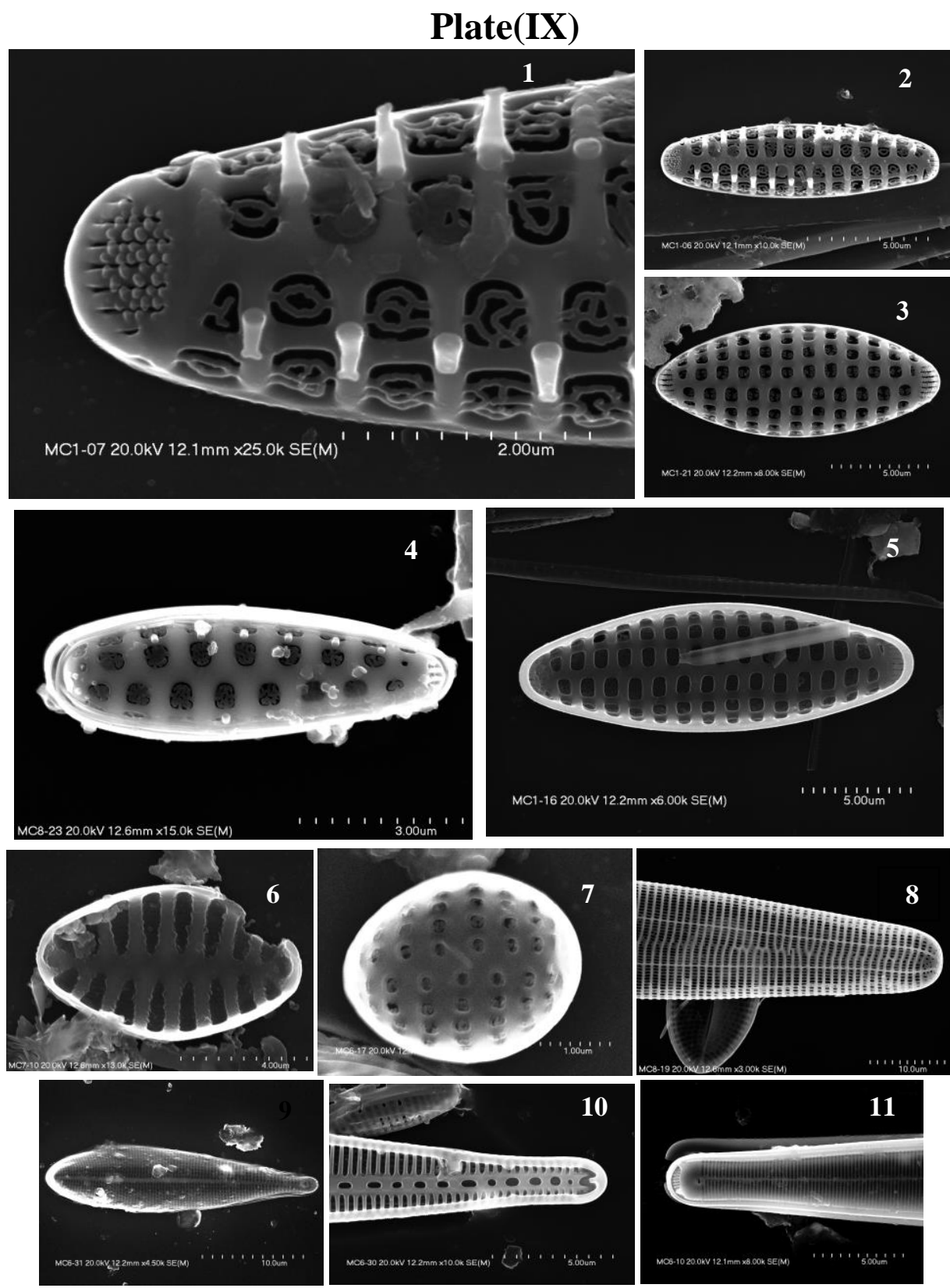

Figures 1-11; 1-3 Fragilaria pinnata v. pinnata Ehr., 4- F. berolinensis (Lemm.) Lange-Bertalot, 5- F. leptostauron v. dubia (Grun.) Hust., 6- F. construens f. subsalina (Hust.) Hust., 7- F. sopotensis Witkowski \& Lange- Bertalot, 8- Ardissonia formosa

(Hantzsch) Grun. 9, 10- Licmophora gracilis v. anglica (Kütz.) Peragallo, 11Hyalosynedra laevigata (Grun.) Williams \& Round. 
Plate $(\mathrm{X})$
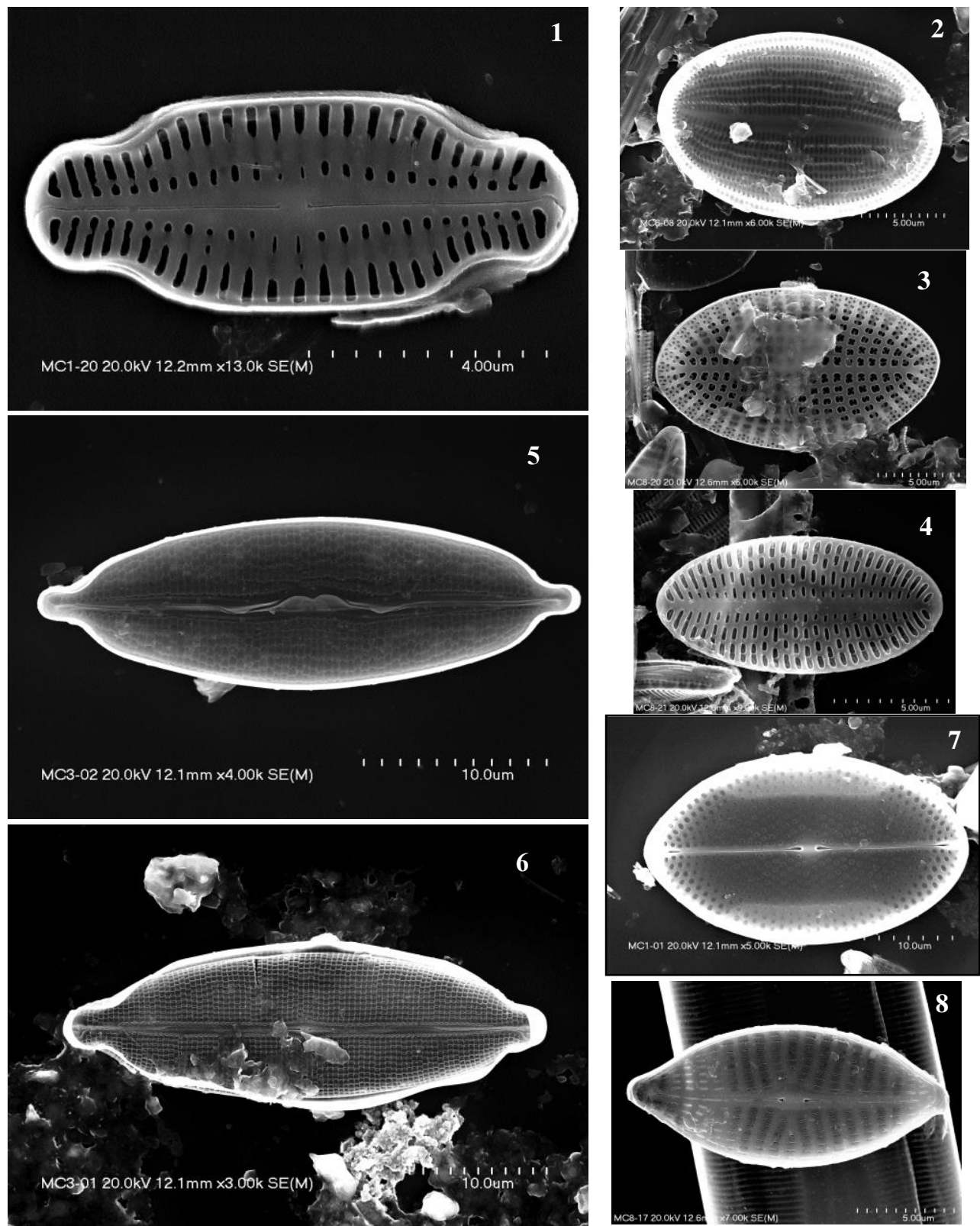

Figures 1-8; 1- Achnanthes amoena Hust., 2-Cocconeis krammerii Lange-Bertalot \& Metzeltin, 3- C. scutellum v. speciosa (Greg.) Cl., 4- C. hauniensis Witkowski, 5Mastogloia erythrea v. erythrea Grun., 6- Craticula cuspidata (Kütz.) D.G. Mann, 7Mastogloia binotata (Grun.) Cl., 8- Fogedia geisslerae Witkowski, Metzeltin \& Lange-Bertalot. 
Preliminary Study on The Diatom Flora of Coastal Periphitic Assemblages of .......

\section{Plate (XI)}
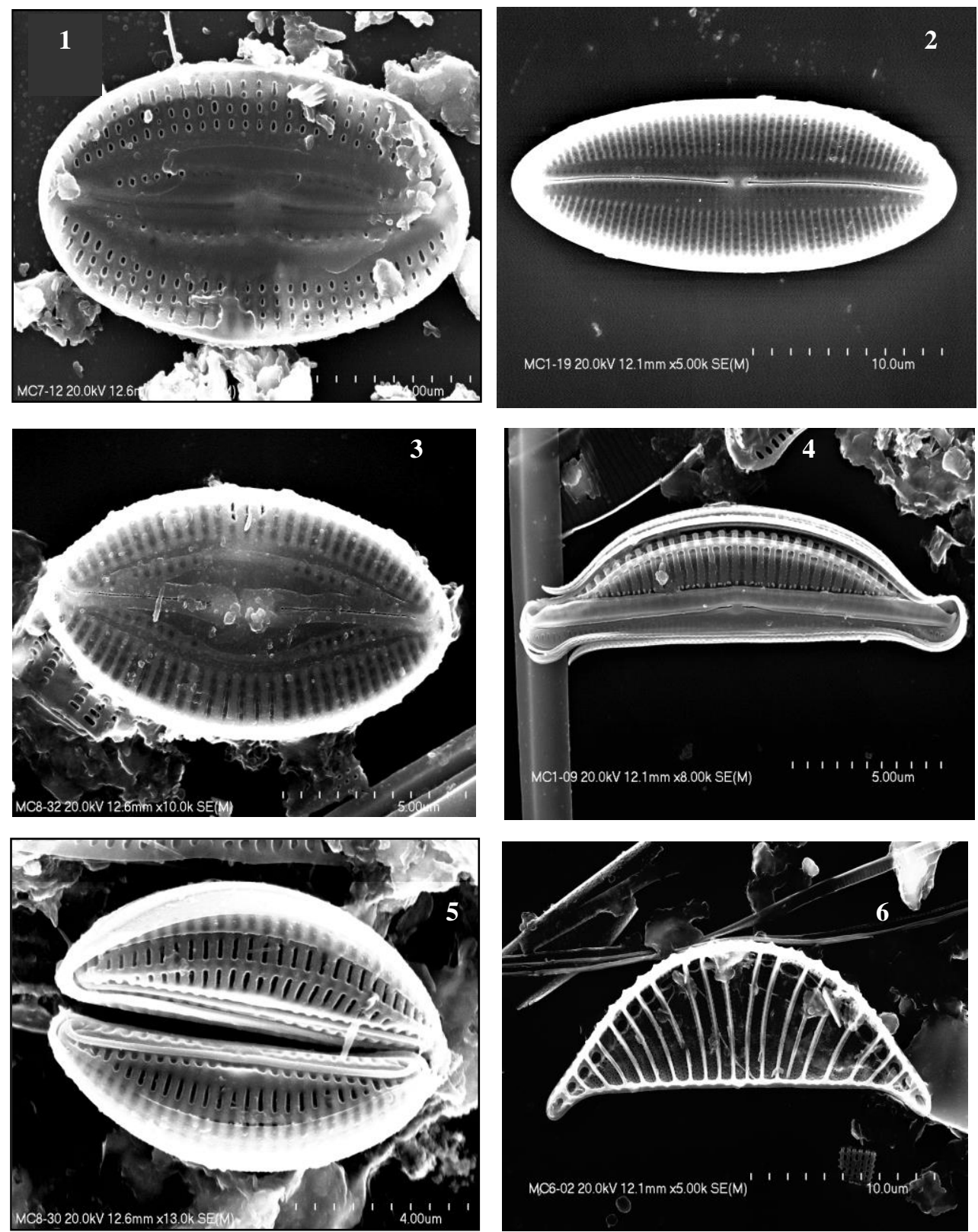

Figures 1-6; 1- Fallcia tenera (Hust.) D.G. Mann, 2- F. litoricola (Hust.) D.G. Mann, 3- F. florianae (Moeller) Witkowski, 4- Amphora delicatissima Krasske, 5- A. exciliata Giffen, 6- Rhopalodia pacifica Krammer. 


\section{Plate (XII)}
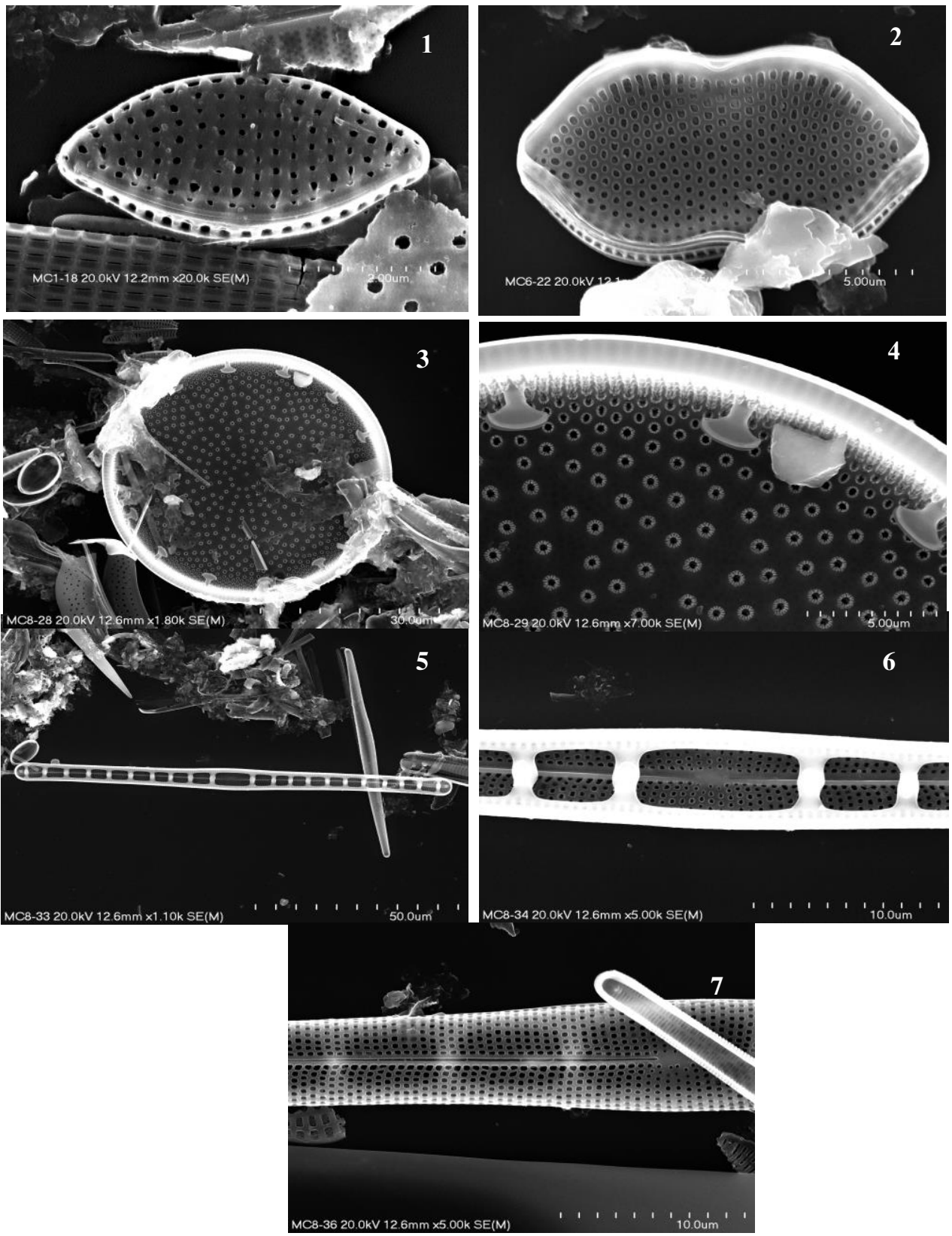

Figures 1-7; 1- Nitzschia dealpina Lange Bertalot \& Hof-mann, 2- N. panduriformis v. continua Grun., 3, 4- unknown diatom no. 1; 3- entire valve, 4- enlarged portion, 57 , unknown diatom no.2 5, whole cell, 6,7 frustule middle part from inferior and exterior, respectively. 